\title{
Contextual effects in vowel perception I: Anchor-induced contrast effects
}

\author{
JAMES R. SAWUSCH and HOWARD C. NUSBAUM \\ State University of New York at Buffalo, Buffalo, New York 14226
}

\begin{abstract}
Results from recent experiments using a selective adaption paradigm with vowels have been interpreted as the result of the fatigue of a set of feature detectors. These results could also be interpreted, however, as resulting from changes in auditory memory (auditory contrast) or changing response criteria (response bias). In the present studies, subjects listened to vowels under two conditions: an equiprobable control, with each of the stimuli occurring equally often, and an anchoring condition, with one vowel occurring more often than any of the others. Contrast effects were found in that vowel category boundaries tended to shift toward the category of the anchor, relative to the equiprobable control. Results from these experiments were highly similar to previous selective adaptation results and suggest that neither feature detector fatigue nor response criterion changes can adequately account for the adaptation/ anchoring results found with vowels.
\end{abstract}

Theories of speech perception have proposed a number of diverse procedures for recovering invariant phonetic information from the continuously varying speech signal. Among these have been theories proposing a direct link between production and perception such as motor theory (Liberman, Cooper, Harris, \& MacNeilage, 1962; Liberman, Cooper, Harris, MacNeilage, \& Studdert-Kennedy, 1967) and analysis by synthesis (Stevens \& Halle, 1967) and more passively oriented stage theories (Fant, 1967). A number of recent theories have been proposed within an information processing framework in which information in the speech signal is transformed and recoded by a number of stages of processing (Cutting, 1976; Pisoni, 1975b; Pisoni \& Sawusch, 1975; Sawusch, 1977a, Tartter \& Eimas, 1975). Within these information procession models, two questions can be addressed. The first asks what the levels of processing between input (speech waveform) and output (phonetic code) are, and the second asks what operations occur within each level.

As one possible answer to the second question, feature detectors have recently received a great deal of attention in speech perception. The primary evidence supporting the role of feature detectors in speech perception has come from studies using the selective adaptation paradigm (see Ades, 1976; Eimas \& Miller, 1978). Briefly, this paradigm involves pre-

The research reported here was supported by SUNY Research Foundation and University Funds grants. The authors would like to thank Dr. David B. Pisoni for making the facilities at Indiana University available for generating the audiotapes and Eileen Schwab for her assistance in running subjects and thoughtful comments on an earlier draft. Reprint requests should be sent to the first author at the Department of Psychology, 4230 Ridge Lea Road, Buffalo, New York 14226. senting the subject with repeated occurrences of an adapting sound and then testing the subject's identification (or discrimination) of a set of stimuli. These results are then compared to the same subject's unadapted, baseline identification (discrimination) of the same stimuli. The typical result has been that the category boundary for the test series shifts following adaptation. Figure 1 summarizes the type of effect that is typically found. The solid line represents the subject's rating function for the stimulus set before adaptation (baseline). When Stimulus 1 (arrow on left) is used as an adaptor, the rating function shifts toward the Stimulus 1 end of the series (function on left). Similarly, when Stimulus 7 is used as an adaptor, the function shifts toward Stimulus 7 (on the right).

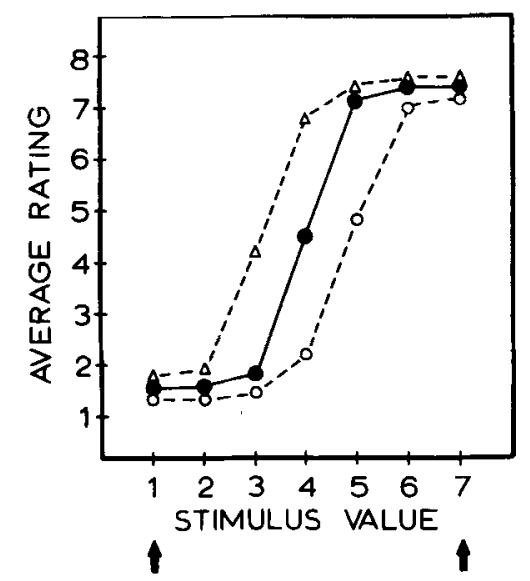

Figure 1. Typical baseline (solid), post Stimulus 1 adaptation (open triangles) and post Stimulus 7 adaptation (open circles) rating functions. 
This type of result has been found for stopconsonant stimuli varying along the phonetic features of voicing (Eimas, Cooper, \& Corbit, 1973; Eimas \& Corbit, 1973), place of articulation (Bailey, 1975; Cooper, 1974; Diehl, 1975; Sawusch, 1977a; Tartter \& Eimas, 1975), and manner (Bailey, 1975; Cooper, Ebert, \& Cole, 1976; Diehl, 1976). These adaptation results have generally been interpreted as supporting the existence of feature detectors for certain auditory components in the speech signal (see Ades, 1976; Cooper, 1975; Eimas \& Miller, 1978, for reviews). These feature detectors may respond to various spectral or temporal patterns in the speech signal (see Blumstein, Stevens, \& Nigro, 1977; Diehl, 1976; Pisoni \& Tash, 1975; Sawusch, 1977a, 1977b).

Besides feature detector fatigue, two other interpretations of category boundary shifts in selective adaptation experiments have emerged. Simon and Studdert-Kennedy (1978) have proposed an auditory contrast explanation in which an adaptor establishes an "auditory ground" that subsequent stimuli are compared with. Their interpretation is based on a comparison of selective adaptation and anchoring procedures. In their anchoring experiments, category boundaries for various sets of stimuli were compared across two conditions: an equiprobable control and an anchoring series in which one (endpoint) stimulus occured more often than the other stimuli. Simon and Studdert-Kennedy found that the results of anchoring and adaptation procedures were often very similar, in that the category boundary for a set of stimuli would shift toward the more frequently occurring category (adaptor/anchor). Simon and Studdert-Kennedy (1978) concluded that feature detector fatigue was not a sufficient explanation for the adaptation and anchoring results, and proposed the auditory contrast explanation (but see Sawusch \& Pisoni, Note 1, for an alternative interpretation of speech anchoring results).

This interpretation is also consistent with a number of other experiments that have shown contextual influences in speech perception (Broadbent \& Ladefoged, 1960; Eimas, 1963; Fry, Abramson, Eimas, \& Liberman, 1962; see Simon \& StuddertKennedy, 1978, for review). Recent experiments reported by Brady and Darwin (1978), Diehl, Elman, and McCusker (1978), and Elman (Note 2) are also consistent with the auditory contrast explanation. Although Diehl et al. (1978) have referred to their explanation as "response contrast," they have also referred to this response contrast as occurring at an auditory level of processing (similar to Simon \& StuddertKennedy, 1978). Consequently, the term "auditory contrast" seems to be more appropriate.

The auditory contrast explanation outlined above, is, in many respects, similar to adaptation level theory as proposed by Helson (1964). In adaptation level theory, previous stimuli would be combined to form an adaptation level against which new stimuli would be compared. If the assumption is made that the adaptation level occurs at an auditory level of processing, then an adaptation level theory explanation of selective adaptation and anchoring results is very similar to the auditory contrast explanation just described (but see Simon \& Studdert-Kennedy, 1978, for an alternative view). Since both adaptation level theory and the auditory contrast explanation interpret changes in phonetic category boundaries as due to changes in a perceptual referent, these two explanations will be grouped toegether here under the term "auditory contrast."

The third interpretation that has emerged for selective adaptation results is that of a response bias on the part of subjects (Rosen, Note 3). Subjects, having heard an adapting syllable repeated many times, may simply use categories other than that of the adaptor in responding. In particular, Rosen has interpreted the results of a number of anchoring experiments as supporting a range-frequency theory (Parducci, 1963, 1965, 1975) explanation of selective adaptation effects. Although range-frequency theory was originally proposed to describe the mapping of arbitrary categories onto equally discriminable stimuli, it may be extendable to the nonarbitrary categories and the unequally discriminable sounds of speech. However, for our purposes, the most important aspect about range-frequency theory is that it claims that changes in identification functions are the result of modifications in labeling strategies (response bias) and are not due to changes in a perceptual referent. Thus, the range-frequency theory interpretation of the category boundary shifts in adaptation/anchoring experiments is in direct conflict with both the feature detector fatigue and auditory contrast explanations with regard to the level(s) of processing involved. (See also Simon \& Studdert-Kennedy, 1978; Sawusch \& Pisoni, Note 1, for other evidence and views on the efficacy of a range-frequency theory interpretation of adaptation/anchoring results.)

Current evidence seems to favor the feature detector fatigue and auditory contrast explanations of selective adaptation and anchoring results with stop consonants (see Eimas \& Miller, 1978; Simon \& StuddertKennedy, 1978; Sawusch \& Pisoni, Note 1). However, all three alternatives need to be considered with regard to selective adaptation results that have been reported for a set of isolated, steady-state vowels (Morse, Kass, \& Turkienicz, 1976). In their experiments, Morse et al. used a set of 13 synthetic vowels which varied from [i] (as in beet) through [I] (as in bit) to $[\varepsilon]$ (as in bet). Stimuli 1 ([i]), 7 ([I]), and 13 ([ $\varepsilon])$ were used as adaptors. However, the results of the Morse et al. experiment differed from the usual findings of experiments using stop consonants. In particular, two aspects of their results seem to be 
crucial. One is that Stimulus 7, the [I] adaptor, had no consistent effects on subjects' responses to the test series. This is one of the few times that a speech adaptor, drawn from a syllable test series, had little effect on the test series. The second aspect of Morse et al.'s results that is not consistent with stop consonant results is that the [i] adaptor (and the [ $\varepsilon$ ] adaptor) had an effect at both the [i]-[I] category boundary and the $[\mathrm{I}]-[\varepsilon]$ category boundary. Cooper (1974) had found that for a [ba]-[da]-[ga] place series, an adapting syllable affected only the adjacent category boundary. (See also Foreit, 1977, for similar results using Thai speakers and a series of stops varying along the voicing dimension.) Morse et al. (1976) interpreted their vowel adaptation results as resulting from the fatigue of a set of relative feature detectors. However, given the differences between the vowel adaptation results of Morse et al. and previous adaptation studies with stop consonants and other wellestablished differences between stop consonant and vowel perception, this conclusion may be premature. In particular, vowel and stop consonant perception seem to differ in two crucial respects: the role of auditory memory in vowel perception and the tremendous influence of contextual information on vowel perception.

A number of studies have shown that the free recall of stop consonants and vowels differs. Crowder (1971, 1973) has shown that for immediate recall of lists of syllables which differ only in their vowels, recency, modality, and suffix effects are found. That is, the last syllables presented are recalled better (recency effect), auditory presentation of syllables yields better recall than visual presentation (modality effect), and presentation of a post list item interferes with recall of the items at the end of the list (suffix effect). Crowder $(1971,1973$; also Cole, 1973) found these effects for vowels, but found no such effects for lists of syllables varying only the stop consonants. Cole (1973) and Crowder $(1971,1973)$ have explained their results in terms of differences in auditory memory between vowels and stop consonants. Crowder has further suggested that auditory information about vowels is retained in precategorical acoustic storage (PAS, Crowder \& Morton, 1969) while auditory information about the stops is not retained in the PAS. A similar explanation has been offered by Fujisaki and Kawashima $(1969,1970)$ and Pisoni $(1973,1975 a)$ for differences between the categorical perception of stop consonants and the continuous perception of vowels. Thus, the effects observed by Morse et al. (1976), in their adaptation experiment with vowels, could have been due to influences of the adaptor on auditory memory (PAS) for their vowel test items. Specifically, at the end of a sequence of the adapting vowel, PAS would contain only information pertaining to the adapting vowel. If subjects identify the test vowels with reference to the information in PAS (cf. ABX discrimination results, Pisoni, 1973, 1975a), then vowel identification would show contextual effects. Comparison of the test items with PAS might accentuate the differences between the test item and the adaptor. This would lead to the classification of vowels that are relatively ambiguous into categories further away from the adaptor (a contrastive change). This explanation hinges, of course, on whether or not vowels show contrast effects (change of ambiguous vowels into categories other than that of the context).

Contextual information has been shown to be tremendously influential in vowel perception. Eimas (1963) and Fry, Abramson, Eimas, and Liberman (1962) have shown that vowels do indeed exhibit contrast effects. These investigators had subjects identify vowels that were presented in triads. Their results showed that the identification of any particular vowel tended to migrate toward categories other than that of the vowels with which it was grouped in the triad. This effect was especially pronounced for the relatively ambiguous vowels in their test series. In a similar vein, Broadbent and Ladefoged (1960; Broadbent, Ladefoged, \& Lawrence, 1956) have shown that the formant frequencies of a precursor phrase will influence the perception of an ambiguous synthetic word as either "bit" or "bet." The effect that they found was also one of contrast. That is, the ambiguous word was identified as belonging to the category that was different from (contrasted with) the spectral information in the precursor phrase. Broadbent and Ladefoged (1960) interpreted their results in terms of a modified version of adaptation level theory (see Helson, 1964). Thompson and Hollien (1970) found a similar contrast effect. In their experiment, subjects identified the second of a pair of synthetic vowels. When the second vowel was "ambiguous" (had formant frequencies between those of "good examples" of various vowels), the subjects displayed a tendency to use categories other than that of the precursor vowel in identifying the second vowel (results very similar to Fry et al., 1962). Since the adaptation results found by Morse et al. (1976) for vowels can also be described as a contrast effect (ambiguous items near the category boundary fall into the adjacent or contrasting category after adaptation), the same processes that underlie the results of Broadbent and Ladefoged (1960), Fry et al. (1962), Eimas (1963), and Thompson and Hollien (1970) may be responsible for the adaptation results observed for vowels.

Recently, Sawusch and Pisoni (Note 1) have used an anchoring paradigm to investigate the perception of stop consonants, vowels, and other auditory stimuli. Using a set of synthetic, steady-state vowels varying from [i] to [I], subjects were run in two conditions. In the control condition, each vowel occurred equally often. In the anchoring conditions, one of the end-point vowels occurred four times as often as each 
of the other vowels. Sawusch and Pisoni (Note 1) found that the category boundary between [i] and [I] moved toward the category of the anchoring vowel, relative to the equiprobable control. Simon and Studdert-Kennedy (1978) have reported a similar result for a [bae]-[d $\varepsilon] C V$ series. These contrast effects are very similar to some of the category boundary shifts found by Morse et al. (1976) using the selective adaptation procedure. Simon and StuddertKennedy interpreted their vowel anchoring results as due to auditory contrast, as described earlier. Sawusch and Pisoni attributed their vowel anchoring results to the same processes that mediate the continuous perception of vowels. From the work of Crowder (1971, 1973) and Pisoni $(1973 ; 1975 a)$, this would seem to be changes in auditory memory. In particular, each stimulus may be categorized by a comparison with auditory memory of the immediately preceding stimuli as well as long-term memory prototypes for the relevant categories. Auditory memory would contain precategorical information about previous vowel stimuli, such as formant frequencies or some ratio or difference between formant frequencies (cf. Gerstman, 1968). In a standard identification sequence, each stimulus occurs equally often in random order. Thus, in the subject's identification of any particular stimulus, information from every stimulus has an equal probability of being present in auditory memory and available for comparison. By comparison, in an adaptation or anchoring procedure, one stimulus, the adaptor/anchor, is presented more often than the other stimuli and is thus more likely to be present in auditory memory when any particular stimulus is identified. Thus, the anchor contributes disproportionately to the "auditory ground" (cf. Simon \& Studdert-Kennedy, 1978) and ambiguous, boundary stimuli now contrast with this ground and are placed in the category opposite (away from) the anchor. This description is very similar to a version of adaptation level theory proposed by Restle (1978). In this version of adaptation level theory, three factors contribute to the adaptation level and thus to the judgment of a stimulus: the stimulus itself, the immediate context, and the background (usually held constant). In terms of the present description of auditory contrast, the immediate context is provided by the information in auditory memory about the immediately preceding stimuli and the background is provided by a set of internal referents or prototypes in long-term memory. Thus, auditory ground is synonymous with adaptation level, and this conceptualization of adaptation level theory can account for the category boundary shifts found in both adaptation and anchoring experiments.

Thus, at this point, three possible explanations for the previous vowel adaptation results have been outlined: (1) the fatigue of a set of feature detectors (Morse et al., 1976); (2) changes in auditory memory
(Sawusch \& Pisoni, Note 1) where adaptation level theory may provide a quantitative description of these changes; and (3) a change in labeling induced by a response bias where range-frequency theory may describe the results. The present set of experiments was intended to explore these various alternative explanations of selective adaptation with vowels and to explore the similarities and differences between adaptation and anchoring.

\section{EXPERIMENT 1}

The first experiment was undertaken to extend the anchoring procedure of Sawusch and Pisoni (Note 1) to the full 13 vowel series used by Morse et al. (1976). In this procedure, one vowel occurs more often than each of the other vowels. If these vowels are widely spaced in time and if the extra occurrences of the more frequently occurring vowel (anchor) are interspersed among the rest of the vowels, then it is very unlikely that any feature detectors would be fatigued. Rather, fatigue would seem to depend upon close, repeated presentation of certain critical information. These conditions do not occur in the anchoring procedure as used by Sawusch and Pisoni (Note 1). Thus, if we obtain results similar to the category boundary shifts found by Morse et al. (1976), using the same 13 vowels in an anchoring procedure, we will have evidence that is inconsistent with a feature detector fatigue interpretation.

\section{Method}

Subjects. The subjects were 33 undergraduates at SUNY/Buffalo who participated as part of a course requirement. None of the subjects had previously participated in a speech experiment. All subjects were right-handed native speakers of English with no reported history of either speech or hearing disorder.

Stimuli. The same 13 vowel set used by Morse et al. (1976) was used in this experiment. These vowels were originally generated by Pisoni (Note 4) on the vocal tract analogue synthesizer at the Research Laboratory of Electronics, Massachusetts Institute of Technology. These stimuli varied in their formant frequencies for their first three formants. Formant frequencies for this series are shown in Table 1. All of the vowels were steady state (no change in formant frequencies over time) and $300 \mathrm{msec}$ in duration. A complete description of these vowel stimuli can be found in Pisoni (Note 4). These 13 vowels were recorded on audiotape and then digitized using the PDP-11 computer in the Speech Perception Laboratory at Indiana University. These stimuli were then reconverted into analogue form and recorded to make eight test tapes. Two of these tapes were control tapes. On each of these tapes, the 13 stimuli occurred five times each in random order. The two [i] anchor tapes each contained 40 presentations of Stimulus 1 ([i]) and 5 of each of the other 12 stimuli in random order. The two [I] and two $[\varepsilon]$ anchor tapes were contructed in a similar manner with $\mathbf{4 0}$ occurrences of Stimulus 7 ([I]) or Stimulus $13([\varepsilon])$ and 5 of each of the other 12 stimuli in random order. However, the order of stimuli was constrained such that no single stimulus ever occurred more than three times in succession. All tapes were recorded with $4 \mathrm{sec}$ between stimuli.

Procedure. The subjects were divided into three groups of 11 subjects each. They were run in small groups of from four to seven 
Table 1

First, Second, and Third Formant Frequencies for the Vowel Stimuli

\begin{tabular}{cccc}
\hline Stimulus & $F_{1}$ & $F_{2}$ & $F_{3}$ \\
\hline 1 & 270 & 2300 & 3019 \\
2 & 285 & 2262 & 2960 \\
3 & 298 & 2226 & 2902 \\
4 & 315 & 2180 & 2836 \\
5 & 336 & 2144 & 2776 \\
6 & 353 & 2103 & 2719 \\
7 & 374 & 2070 & 2666 \\
8 & 397 & 2032 & 2628 \\
9 & 420 & 1999 & 2581 \\
10 & 444 & 1966 & 2556 \\
11 & 472 & 1926 & 2544 \\
12 & 501 & 1898 & 2518 \\
13 & 530 & 1858 & 2492 \\
\hline
\end{tabular}

Note-Taken from Pisoni, (1971).

subjects at a time. Each subject participated in two 1-h sessions on successive days. The stimulus tapes were reproduced on a Revox A-700 tape deck and presented binaurally to subjects at an intensity of $80 \mathrm{~dB}$ SPL (for the Stimulus 1 vowel [i]) via Telephonics TDH-39 matched and calibrated headphones. Each group listened to the two control tapes at the beginning of each session. The subjects were informed that they would be listening to synthetic vowels that would sound like the [i] in beet, the [I] in bit, or the $[\varepsilon]$ in bet. They were asked to identify each vowel as [i], [I], or $[\varepsilon]$ and write down their responses in prepared booklets. The subjects were also asked to give a rating response after each identification response which indicated how sure they were that they had identified the vowel correctly. A 4-point scale was used with a 1 indicating that the subject was positive his (her) identification was correct, a 2 indicating a probable correct, a 3 indicating a possible correct, and a 4 indicating a guess.

Following the control tapes, each of the three groups listened to a different set of anchor tapes (the same anchor on both days). The subjects were not given any new instructions concerning these tapes. They used the same three category-plus-rating response set. By the end of the experiment, each subject had provided at least 20 responses to each of the $\mathbf{1 3}$ stimuli under both the control and one of the anchor conditions.

\section{Results}

The identification plus rating responses were converted into an 8-point rating scale, with a rating of 1 indicating an extremely confident response for a particular category and an 8 indicating extreme confidence that the syllable was not in the particular category. The results for the [i] anchor group using this scale are shown in Figure 2. Both the [i]-[I] category boundary and the $[\mathrm{II}]-[\varepsilon]$ category boundary showed significant shifts toward [i] $[\mathrm{t}(10)=9.48, \mathrm{p}<.001$, for the $[i]-[I]$ boundary; $t(10)=3.05, p<.01$, for the [I]- $[\varepsilon]$ boundary]. ${ }^{1}$

The results for the [I] anchor group are shown in Figure 3. No significant shift in the [i]-[1] category boundary was found $[t(10)=.32, p>.25]$. A significant shift in the $[\mathrm{I}]-[\varepsilon]$ category boundary toward [I] was found $[t(10)=4.00, \mathrm{p}<.005]$. The overall tendency in subjects' responses was for the [I] category to shrink. This shrinkage was marginally significant $[\mathrm{t}(10)=2.06, .05<\mathrm{p}<.1$, for the difference between [i]-[I] and [I]-[ $\varepsilon]$ category boundaries before and after anchoring]. This exactly parallels the results of Morse et al. (1976), who also found a marginally significant shrinkage of the [I] category following adaptation with [I] (Stimulus 7).

The $[\varepsilon]$ anchor results are shown in Figure 4. Both category boundaries showed a significant shift toward the $[\varepsilon]$ category $[t(10)=2.07, p<.05$, and $t(10)$ $=3.45, \mathrm{p}<.005$, for the [i]-[I] and $[\mathrm{I}]-[\varepsilon]$ category boundaries, respectively]. The mean boundary shifts from the present experiment and the adaptation experiment of Morse et al. (1976) are shown together in Table 2. Although the absolute magnitudes of the boundary shifts differ, the patterns of results are markedly similar.

\section{[i] Anchor Group}

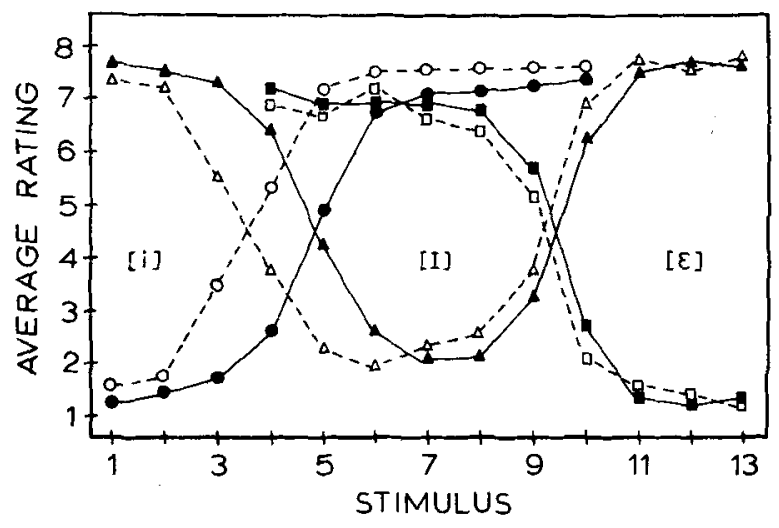

Figure 2. Baseline (solid) and [i]-anchored (dashed) rating functions for the [i] anchor group: [i] (circles), [I] (triangles), and [E] (squares) rating functions are shown separately ([i] ratings for Stimuli 11-13 and [ $\varepsilon$ ] ratings for Stimuli 1-3 omitted).

\section{[I] Anchor Group}

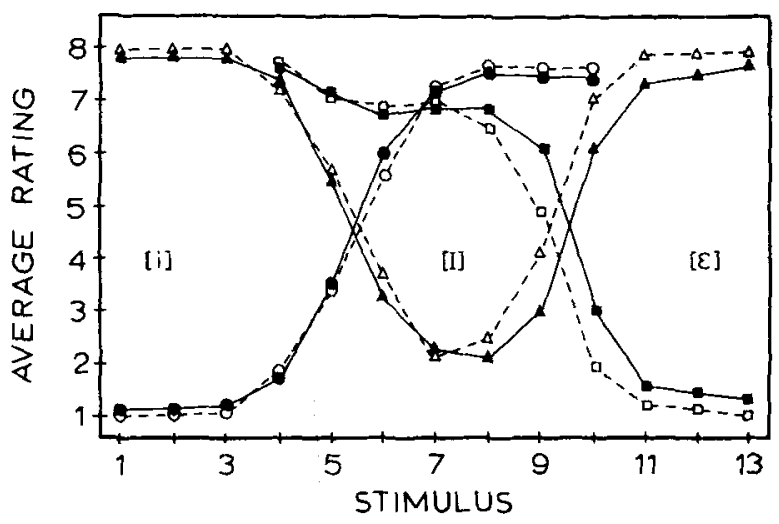

Figure 3. Baseline (solid) and [I]-anchored (dashed) rating functions for the [I] anchor group: [i] (circles), [I] (triangles), and [E] (squares) rating functions are shown separately ([i] ratings from Stimuli 11-13 and [ $\varepsilon$ ] ratings from Stimuli 1-3 omitted). 
[E] Anchor Group

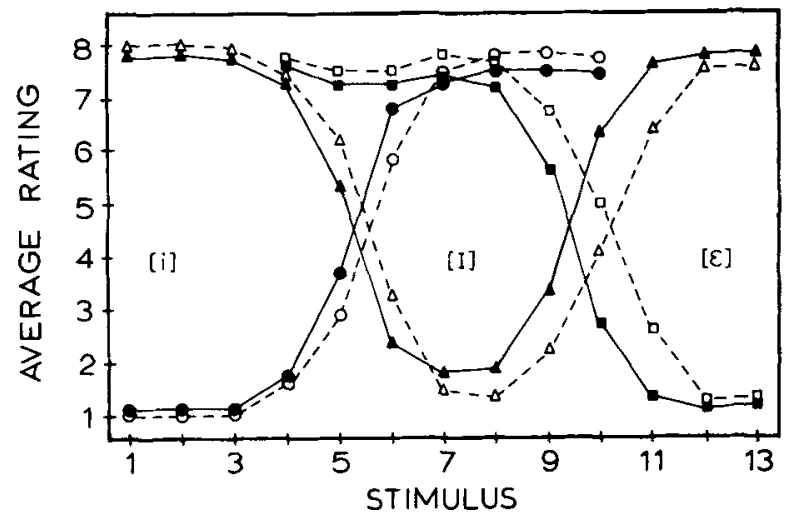

Figure 4. Baseline (solid) and [ $\varepsilon]$-anchored (dashed) rating functions for the [ $\varepsilon$ ] anchor group: [i] (circles), [I] (triangles), and [E] (squares) rating functions are shown separately (ii] ratings for Stimuli 11-13 and [ $\varepsilon$ ] ratings for Stimuli 1-3 omitted).

\section{Discussion}

The results of Experiment 1 and Morse et al. (1976) indicate that anchoring and adaptation with vowels produce similar patterns of results. Although the overall shifts in category boundaries were somewhat smaller for anchoring, this may have been due to the basic difference between adaptation and anchoring procedures. Morse et al. (1976) presented their adaptors repeatedly for $1 \mathrm{~min}$ (100 presentations) for every set of 13 test stimuli. In the present anchoring procedure, 35 extra presentations of the anchor were mixed in with a set of 65 test stimuli. This difference in number of adaptors vs. anchors may account for the overall smaller shifts in the present experiment. Thus, it seems reasonable to conclude that adaptation and anchoring affect the same level(s) of vowel processing and have similar effects at this level (or levels). This equivalence between anchoring and adaptation procedures runs counter to the feature detector fatigue explanation offered by Morse et al. (1976). The wide spacing between extra occurrences of the anchoring vowel makes fatigue of feature detectors (relative or otherwise) quite unlikely.

Recently, Simon (Note 5) has reported the results of an adaptation experiment in which the interval between adaptor repetitions was varied. Her results indicate significant category boundary shifts with $1,750 \mathrm{msec}$ between stimuli (using 16 repetitions of the adaptor before each block of test trials). If one accepts the feature detector fatigue explanation, then these results might lead one to expect that fatigue may still occur with $4 \mathrm{sec}$ between stimuli. However, Simon's results also show that the optimal adaptor repetition interval was around $750 \mathrm{msec}$, which is substantially less than $4 \mathrm{sec}$. Secondly, in Experiment 1, the extra presentations of the anchor were widely interspersed with other stimuli. Even if other stimuli very similar to the anchor (within 2 stimulus units) are counted with it, there were still no substantial runs (greater than seven occurrences) of "equivalent" stimuli in these test orders, and even these runs always maintained the $4 \mathrm{sec}$ between stimuli spacing. Thus, we feel that feature detector fatigue is at best a highly improbable explanation of these vowel anchoring results.

This leaves two alternative explanations for the anchoring and adaptation effects found with vowels: changes in auditory memory (auditory contrast) or changes in labeling (response bias). However, before proceeding with a test of these two possibilities, a couple of differences between these results and those of Morse et al. (1976) and Sawusch and Pisoni (Note i) should be noted. First, Morse et al (1976) found no adaptation effect on either the [i]-[I] category boundary or the $[\mathrm{I}]-[\varepsilon]$ boundary using the $[\mathrm{I}]$ adaptor. In the present experiment, the [I] anchor did yield a significant shift in the $[\mathrm{I}]-[\varepsilon]$ category boundary toward [I]. However, Morse et al. did find a trend for the $[\mathrm{I}]-[\varepsilon]$ boundary to move toward [I] that was not significant (see Table 2). In addition, a marginally significant shrinkage in the [I] category following [I] adaptation/anchoring was found in both Morse et al. and Experiment 1.

The second item to note is that Sawusch and Pisoni (Note 1) found a shift in the [i]-[I] category boundary with an [I] anchor. Both Morse et al. (1976) and Experiment 1 found no such shift. In the experiments of Sawusch and Pisoni (Note 1), only the first 7 vowels of the 13 vowel series were used and the [i]-[I] category boundary in the equiprobable control condition was near the midpoint of their series (4.1 stimulus units). By comparison, the [i]-[I] category boundaries in Morse et al. (1976) and Experiment 1 were at 6.15 and 5.4 stimulus units, respectively. Thus, the [i]-[I] category boundaries of Morse et al. and Experiment 1 may have already been "shifted" into the [I] category, and further exposure to an [I] adaptor/anchor produced no further shift.

\section{EXPERIMENT 2}

If the explanation outlined above for the failure to find an [i]-[I] category boundary shift with an [I] adaptor/anchor is correct, then, using only the first seven vowels of the test series, we should find an [I]

Table 2

Comparison of Magnitudes of Category Boundary Shifts for Anchoring and Adaptation

\begin{tabular}{|c|c|c|c|c|c|c|}
\hline & \multicolumn{2}{|c|}{$\begin{array}{l}\text { [i] Adaptor/ } \\
\text { Anchor }\end{array}$} & \multicolumn{2}{|c|}{$\begin{array}{l}\text { [I] Adaptor/ } \\
\text { Anchor }\end{array}$} & \multicolumn{2}{|c|}{$\begin{array}{c}\text { [e] Adaptor } \\
\text { Anchor } \\
\end{array}$} \\
\hline & {$[\mathrm{i}]-[\mathrm{I}]$} & {$[I]-[\epsilon]$} & [i] $-[\mathrm{I}$ & $I]-[\epsilon]$ & {$[\mathrm{i}]-[\mathrm{I}]$} & {$[\mathbf{I}]-[\epsilon]$} \\
\hline $\begin{array}{l}\text { Adaptation } \\
\text { Anchoring }\end{array}$ & $\begin{array}{l}1.18 \\
1.52\end{array}$ & $\begin{array}{r}1.02 \\
.53\end{array}$ & $\begin{array}{l}.15 \\
.06\end{array}$ & $\begin{array}{l}.60 \\
.47\end{array}$ & $\begin{array}{l}.96 \\
.37\end{array}$ & $\begin{array}{r}1.19 \\
.78\end{array}$ \\
\hline
\end{tabular}

Note-Adaptation data taken from Morse et al. (1976). 
anchor shift (as per Sawusch \& Pisoni, Note 1). Experiment 2 was conducted in part to check this explanation.

The other rationale for Experiment 2 was to investigate one aspect of a response bias explanation of anchoring. In particular, the number of response categories available to the subject should influence the magnitude of change in the category boundary when the range of stimuli is extended (cf. Parducci, 1975). In particular, if an $[\varepsilon]$ anchor is added to an [i]-[I] test series, subjects should show a larger movement of the category boundary toward [I] if they only have two response categories ([i] and [I]) than if they have three response categories $([i],[I]$, and $[\varepsilon])$. This result would occur because, in one case, subjects would be forced to stretch two response categories to cover an increased range of vowel stimuli (cf. range-frequency theory, Parducci, 1965). If this pattern of results is found, then we will have shown that it is possible to induce a labeling change/response bias during vowel identification in an anchoring paradigm.

\section{Method}

Subjects. The subjects in this experiment were $\mathbf{4 0}$ undergraduates at the State University of New York at Buffalo, who participated as part of a course requirement. All were right-handed native speakers of English with no reported history of either speech or hearing disorder.

Stimuli. The stimuli in this experiment were taken from the same set of 13 synthetic steady-state vowels that were used in Experiment 1 . These stimuli were converted to analog form and recorded to make four test tapes.

The control tape contained 10 occurrences of each of the first seven stimuli in random order. The [i] anchor tape contained 40 occurrences of Stimulus 1 ([i]) and 10 occurrences of each of the other six stimuli (Stimuli 2-7). Similarly, the [I] anchor tape contained 40 occurrences of Stimulus 7 and 10 each of Stimuli 1-6. The $[\varepsilon]$ anchor tape contained 40 occurrences of Stimulus $13([\varepsilon])$ and 10 each of Stimuli 1-7 in random order. In all four tapes, the order of stimuli was constrained such that no one stimulus ever occurred more than three times in succession. All tapes were recorded with $4 \mathrm{sec}$ between stimuli.

Procedure. Subjects were divided into four groups of 10 each. They were run in smaller groups of from four to six subjects at a time. The audiotapes were reproduced and presented to the subjects in the same manner as in Experiment 1. All subjects were run for $1 \mathrm{~h}$. At the beginning of their session, all subjects listened to two presentations of the control tape. The subjects were informed that they would be listening to synthetic examples of the vowels [i] as in beet and [I] as in bit and that they were to identify each vowel as either [i] or [I] and write down their responses in prepared response booklets. The subjects were also asked to give a rating response indicating how sure they were that they had identified each vowel correctly. The same 4-point scale used in Experiment 1 was used here.

Following the two control tape presentations, each group heard two presentations of an anchoring tape. The [il-, $[\mathrm{I}]-$, and $[\varepsilon]-$ anchor tapes were each given to one group. These subjects were not given any new instructions about these tapes. They were asked to continue to use the two-category ([i] and [I]) and 4-rating-point response. This $[\varepsilon]$ anchor group will be referred to as the $[\varepsilon]$ as $[I]$ group. The fourth group also listened to the $[\varepsilon]$ anchor tape. However, these subjects were told that 40 occurrences of an $[\varepsilon]$ vowel were going to be added. Further, the occurrences of the $[\varepsilon]$ vowel were premarked on their response sheets. The subjects were asked

to simply check them off when they occurred. These two different $[\varepsilon]$ anchor groups represent the manipulation of the number of response categories available to subjects.

\section{Results}

The two-response category plus 4-point rating were unfolded into an 8-point rating scale with a 1 representing a positive [i] and an 8 representing a positive [I]. The results for the [i] and [I] anchor groups are shown in Figure 5 on the left and right sides, respectively. Both anchoring conditions produced contrast effects. For both groups, a significant shift in the category boundary toward the anchor (relative to the equiprobable control) was found $[\mathrm{t}(9)=5.42, \mathrm{p}<$ .001 , for the [i] anchor; $t(9)=4.00, p<.01$, for the [I] anchor]. The average boundary shifts were .77 stimulus units toward [i] for the [i] anchor group and .42 units toward [I] for the [I] anchor group. These results replicate the earlier findings of Sawusch and Pisoni (Note 1).

The results of the two $[\varepsilon]$ anchor groups are shown in Figure 6. On the left is the group which was informed about the 40 added $[\varepsilon]$ vowels. A significant shift in the category boundary was found $[t(9)=$ $6.89, p<.001]$. The magnitude of this shift was almost double that found with the [I] anchor (.81 vs. .42 units) and was significantly greater than the [I] anchor shift $[t(18)=2.42, p<.05$, for a two-tailed, independent $t$ test]. On the right are the results for the $[\varepsilon]$ anchor group, which was not informed about the addition of the new $[\varepsilon]$ vowel in the anchor tapes (labeled $[\varepsilon]$ as [I]). This group also showed a significant movement of the category boundary $[\mathrm{t}(9)=$ $8.21, p<.001]$. The mean shift of 1.78 stimulus units was significantly larger than both the [I] anchor group shift and the other $[\varepsilon]$ anchor group shift $[\mathrm{t}(18)=$ $5.62, \mathrm{p}<.001$, and $\mathrm{t}(18)=3.95, \mathrm{p}<.002$, respectively, for the two-tailed independent $t$ tests]. Of the 10 subjects in the $[\varepsilon]$-as-[I] anchor group, 4 exhib.

[i] Anchor

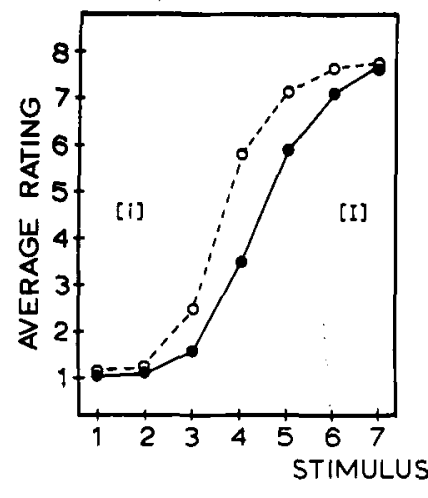

Figure 5. Baseline (solid) and anchored (open-dashed) rating functions for the [i] anchor (left) and [I] anchor (right) groups in Experiment 2. 


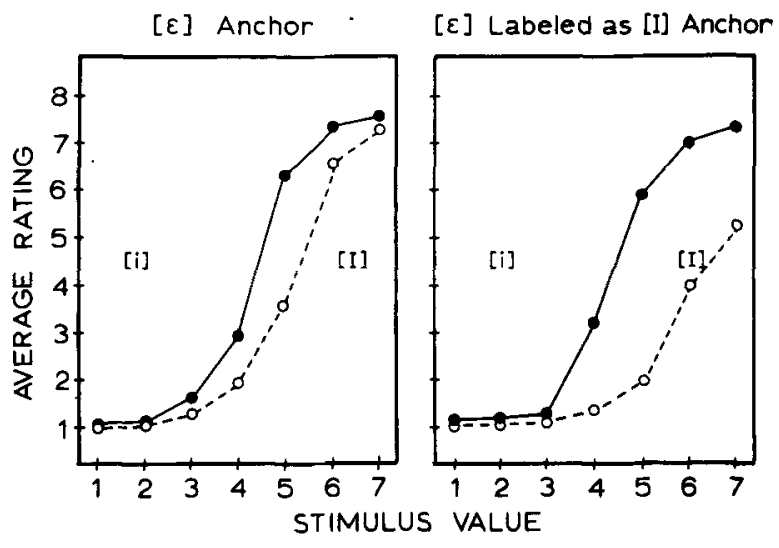

Figure 6. Baseline (solid) and anchored (open-dashed) rating functions for the three-response category $[\varepsilon]$ anchor group (left) and the two-response category $[\varepsilon]$ as [I] anchor group (right) in Experiment 2.

ited a rather drastic change in their identification of the seven test vowels. These four subjects identified all seven of the test vowels on the $[\varepsilon]$-as-[I] anchor tape as [i]. This result made it impossible to determine a precise category boundary for these subjects. Consequently, Stimulus 7 was taken as the location of the category boundary for these four subjects. Each of the other subjects showed a distinct category boundary. However, all 10 of these subjects showed decreases in their ratings for Stimuli 4 through $7 .^{2}$

\section{Discussion}

The results of the [i] and [I] anchor groups replicate Sawusch and Pisoni (Note 1). These results demonstrate rather conclusively that an [I] anchor can cause a shift in the [i]-[I] category boundary. It should be noted that the [I]-anchored and the premarked $[\varepsilon]$-anchored category boundaries were at 4.9 and 5.3 stimulus units, respectively. Both of these values are less (nearer the [i] end of the series) than the equiprobable [i]-[I] category boundary in Experiment 1 (5.4 stimulus units). Thus, there do seem to be limited regions within which shifts in category boundaries under anchoring (and adaptation) with vowels can be obtained. In Experiment 1, the [i]-[I] category boundary was apparently near one such limit and no shift was found for an [I] anchor.

The other primary result from Experiment 2 is the difference between the two $[\varepsilon]$ anchor groups. The $[\varepsilon]$ as [I] group, which was forced to use two responses ([i] and [I]) to cover all stimuli, showed a much larger shift in their category boundary than the premarked (three-response category) $[\varepsilon]$ group. These results are consistent with a response bias explanation of vowel anchoring such as range-frequency theory (Parducci, $1963,1965,1975)$, and indicate that discrepancies between the subject's internally available response categories and those that are externally imposed can, within limits, lead to large changes in identification. Thus, part of the anchoring and adaptation effects observed with vowels may be due to a change in labeling induced by a response bias.

\section{EXPERIMENT 3}

At this point, a feature detector fatigue explanation of anchoring and adaptation effects with vowels seems to be untenable. This leaves at least two potential explanations: changes in auditory memory and response bias. The results of Experiment 2 imply response bias in one particular case, where the response categories available to the subject are manipulated. However, this still leaves us with the question of what is happening in the other anchoring conditions, where appropriate response categories are available.

In order to distinguish between changes in auditory memory and response bias, relatively specific predictions from these two explanations are needed. In particular, a more specific response bias model is needed. One relatively simple form of response bias explanation, a probability matching type of explanation, can be tested directly. If the subject is simply trying to use the two (or three) vowel response categories equally often, then the presence of extra members of one category (an anchor) would cause the subjects to shift their category boundary to maintain equal response frequencies. If this were the case, then knowledge of the extra occurrences of one stimulus might be expected to diminish the contrast effect found. However, there was no appropriate control to test this explanation in Experiments 1 or 2. Furthermore, the number of response categories did have a large effect in Experiment 2. This response-categories effect may have been due to a probability matching effect brought about by subjects treating the $[\varepsilon]$ anchor as an [I] vowel in the [ $\varepsilon$ ] as [I] anchor group. Experiiment 3 was intended as a direct test of the probability matching hypothesis, using a situation in which the number of response categories (and range of vowels) was held constant and only instructions were varied. If instructions as to the identity and frequency of the anchor have no effect on anchoring, then the relatively simple probability matching model can be ruled out. On the other hand, since the actual vowels presented to the subjects are identical under the two instructional conditions, the auditory-memory explanation predicts that the same anchoring effects should be found for the different sets of instructions.

\section{Method}

Subjects. The subjects were 32 undergraduates at SUNY/ Buffalo, who participated as part of a course requirement. None of these subjects had participated previously in a speech experiment, and all met the same requirements as previous subjects.

Stimull. The first seven vowels ([i]-[I]) of the 13-vowel set used previously were employed. They were recorded on audiotape to 
make a control tape, an [i] (Stimulus 1) anchor tape, and an [I] (Stimulus 7) anchor tape, as per Experiment 2. The anchoring vowel occurred four times as often as each of the other vowels.

Procedure. The subjects were divided into four groups of eight subjects each. They were run in small groups of from three to five subjects at a time for one 1 -h session. The audiotapes were presented to the subjects as in Experiments 1 and 2. At the beginning of each session, all subjects listened to two presentations of the control tape. The subjects used the same two-category-plus-4-pointrating response procedure that was used in Experiment 2. Following the control tapes, each group listened to two presentations of one of the anchor tapes. Two of the groups listened to the [i] anchor tape, and the other two listened to the [I] anchor tape. Of the two [i] anchor groups, one received the same instructions given to the [i] anchor subjects in Experiments 1 and 2. When the [i] anchor tapes were presented, they were told only that the tape contained $100[i]$ and $[I]$ vowels for them to respond to. The other [i] anchor group received new instructions. They were told that the difference between the previous two $(70$-item) tapes and the next two $(100$ item) tapes was that an extra 30 occurrences of the [i] vowel were randomly distributed throughout each of the next (100-item) tapes. The two [I] anchor groups were similarly given old and new instructions. In all other respects, except instructions, the two [i] anchor groups ([I] anchor groups) were treated identically.

\section{Results}

The identification-plus-rating responses were converted into an 8-point rating scale (as per Experiment 2) with a 1 being a positive [i] and an 8 a positive [I]. The results for the two [i] anchor groups are shown in Figure 7. In both cases, a significant shift in the category boundary toward the anchoring stimulus was found $[t(7)=3.73, p<.01$, for the old instructions; $\mathrm{t}(7)=6.14, \mathrm{p}<.001$, for the new instructions]. The mean shifts of .51 stimulus units for the old instructions and .66 for the new instructions were not significantly different $[t(14)=.90, p>.20$, for a two-tailed, independent $t$ test].

The results for the two [I] anchor groups are shown in Figure 8. Again, a significant category boundary shift toward the [I] anchor was found for both old and new instructions groups $[t(7)=5.21, p<.005$, and $t(7)=3.80, p<.01$, respectively]. The mean shifts of .62 units (old) and .64 units (new) were not signif-
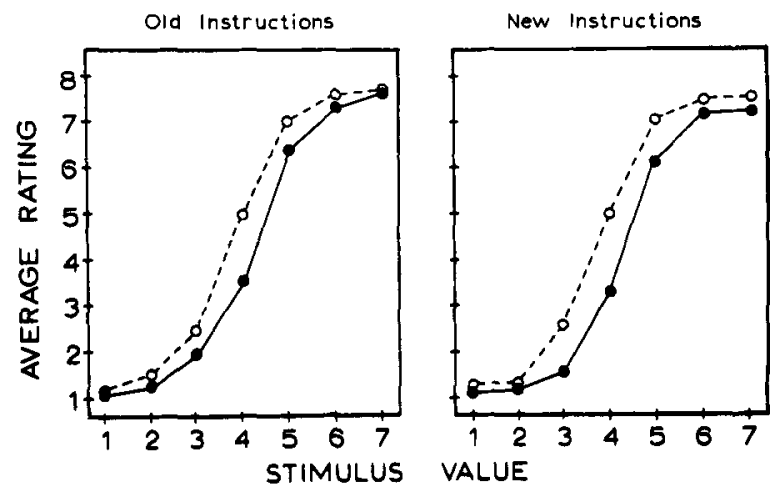

Figure 7. Baseline (solid) and anchored (open-dashed) rating functions for the two [i] anchor groups in Experiment 3: old instructions on the left and new instructions on the right.
[I] Anchor

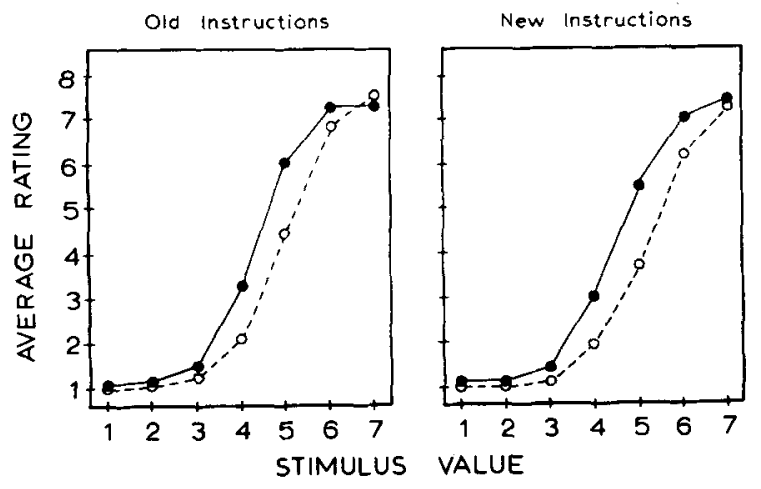

Figure 8. Baseline (solid) and anchored (open-dashed) rating functions for the two [I] anchor groups in Experiment 3: old instructions on the left and new instructions on the right.

icantly different $[t(14)=.11, \mathrm{p}>.80$, for a twotailed, independent $t$ test].

\section{Discussion}

These results clearly demonstrate that instructions had no effect on the vowel identification contrast effects found as a function of anchoring. In particular, knowledge of the extra occurrences of the anchoring vowel did not reduce the size of the contrast effect. In fact, the new instructions groups showed slightly larger effects than the old instructions groups. These results are consistent with those of Broadbent and Ladefoged (1960), who also found that instructions had no influence on the contrast effect with vowels that they observed. This lack of an instructional effect would seem to rule out a relatively simple probability matching explanation of anchoring effects in vowels. Since the range-frequency theory of Parducci $(1965,1975)$ is basically a probability matching theory when stimulus range is held constant and only frequency is varied, these results also cast doubt upon a range-frequency theory interpretation of the vowel anchoring results of Experiments 1 and 3 and the adaptation results of Morse et al. (1976). This leaves us with the auditory memory contrast explanation as the only remaining description that fits all of the data.

\section{GENERAL DISCUSSION}

Earlier, we raised two questions concerning the selective adaptation and anchoring of vowels: the levels of processing that are affected and the type of effect at each level. A secondary question was whether anchoring and adaptation produced the same results. The answer to the secondary question seems to be clear. The nearly identical patterns of results from vowel adaptation (Morse et al., 1976) and vowel anchoring (Experiment 1) indicate that adaptation 
and anchoring probably affect the same levels of processing.

With regard to our primary questions, the results of these experiments favor an auditory contrast interpretation of adaptation and anchoring with vowels. The lack of an instructional effect in Experiment 3 seems to eliminate an overt probability matching type of response bias (and range-frequency theory) as an explanation of anchoring with vowels, at least when the subject has a suitable set of response categories. An explanation in terms of feature detector fatigue seems to be similarly unjustified. In all three experiments, the extra occurrences of a particular vowel (anchor) were widely spaced. Further, the anchoring vowel never occurred more than three times in succession. Thus, it is unlikely that the anchoring vowel fatigued any feature detectors.

These results from the vowel anchoring experiments stand in marked contrast to the results from stopconsonant anchoring and adaptation studies. Although stop CV syllables do show adaptation, they do not show the same degree of anchoring that was found with vowels (see Simon \& Studdert-Kennedy, 1978; Sawusch \& Pisoni, Note 1). The instructions to subjects about the identity of an adapting syllable have also been shown to have no influence on stop CV adaptation (Sawusch \& Pisoni, 1976). However, Experiment 2 clearly showed that if subjects are not told about the addition of an $[\varepsilon]$ vowel and are asked to use just [i] and [I] response categories, a larger shift in the category boundary is found than if they are told about the extra $[\varepsilon]$ vowel. This type of effect is clearly different from results typically found with the stop consonants and probably reflects a labeling change by the subjects. This combined set of results indicates that the processes underlying the anchoring effects found with vowels may be quite different from the adaptation effects found with stop consonants.

Although both the feature detector fatigue and the response bias explanations were found lacking, the auditory contrast explanation involving auditory memory does fit all of the data in Experiments 1 and 2 . In the anchor conditions, the anchoring vowel is the single most common stimulus. Thus, any residual trace of previous stimuli, in auditory memory, is likely to contain more information about the anchoring stimulus than about other stimuli. If identification of each new stimulus is made with reference (contrast) to this memory trace, as well as to some long-term memory prototypes for the relevant categories, then the anchoring conditions should produce changes in identification, especially for otherwise ambiguous (boundary) stimuli. A variation of adaptation level theory (Helson, 1964) proposed by Restle (1978) may piovide a mathematical framework for incorporating the influence of both information from immediately preceding stimuli (auditory memory) and prototypes in long-term memory into one unified auditory ground against which new stimuli are compared. Although this explanation is speculative, it is consistent with much of the data and explanations on PAS (Crowder, 1971, 1973; Crowder \& Morton, 1969) and the continuous perception of vowels (Pisoni, 1973, 1975a).

In summary, the present experiments have provided evidence that results previously obtained using a selective adaptation paradigm with steady-state vowels can be duplicated with an anchoring procedure where the relative frequency of occurrence of various stimuli and/or the range of stimuli used are varied. The results of these experiments argue against either a feature detector fatigue or an overt probability matching explanation of the shifts in category boundaries found with anchoring/adaptation. An adaptation level description of auditory contrast involving auditory memory and internalized referents was proposed which was consistent with the adaptation and anchoring data for vowels.

\section{REFERENCE NOTES}

1. Sawusch, J. R., \& Pisoni, D. B. Anchoring and contrast effects in the identification of speech and nonspeech sounds. Manuscript in preparation.

2. Elman, J. L. Response bias account of selective adaptation. Paper presented at the 94th meeting of the Acoustical Society of America, December 1977, Miami.

3. Rosen, S. M. Range and frequency effects in consonant categorization. Submitted for publication, 1978.

4. Pisoni, D. B. On the nature of categorical perception of speech sounds. Supplement to: Status Report on Speech Research, Haskins Laboratories, November 1971.

5. Simon, H. J. Effects of varying total adaptor energy in selective adaptation. Paper presented at the 94th meeting of the Acoustical Society of America, Miami, December 1977.

\section{REFERENCES}

ADEs, A. E. Adapting the property detectors for speech perception. In R. J. Wales \& E. Walker (Eds.), New Approaches to language mechanisms. Amsterdam: North-Holland, 1976.

BaILEY, P. J. Perceptual adaptation in speech: Some properties of detectors for acoustical cues to phonetic distinctions. Unpublished doctoral thesis, 1975, University of Cambridge, Cambridge, England.

Blumstein, S. E., Stevens, K. N., \& Nigro, G. N. Property detectors for bursts and transitions in speech perception. Journal of the Acoustical Society of America, 1977, 61, 1301-1313.

Brady, S. A., \& DARWIN, C. J. Range effect in the perception of voicing. Journal of the Acoustical Society of America, 1978, $63,1556-1558$.

Broadbent, D. E., \& Ladefoged, P. Vowel judgments and adaptation level. Proceedings of the Royal Society, B, 1960, 151, 384-399.

Broadbent, D, E., Ladefoged, P., \& Lawrence, W. Vowel sounds and perceptual constancy. Nature, 1956, 178, 815-816.

Cole, R. A. Different memory functions for consonants and vowels. Cognitive Psychology, 1973, 4, 39-54.

COOPER, W. E. Adaptation of phonetic feature analyzers for place of articulation. Journal of the Acoustical Society of America, 1974, 56, 617-627.

Cooper, W. E. Selective adaptation to speech. In F. Restle, R. M. 
Shiffrin, N. J. Castellan, H. Lindman, \& D. B. Pisoni (Eds.), Cognitive theory (Vol. 1). Hillsdale, N.J: Erlbaum, 1975.

CoOper, W. E., Ebert, R. R., \& Cole, R. A. Perceptual analysis of stop consonants and glides. Journal of Experimental Psychology: Human Perception and Performance, 1976, 2, 92-104.

Crowder, R. G. The sound of vowels and consonants in immediate memory. Journal of Verbal Learning and Verbal Behavior, 1971, $10,587-590$.

CROWDER, R. G. Representation of speech sounds in precategorical acoustic storage. Journal of Experimental Psychology, 1973, 98, 14-24.

Crowder, R. G., \& Morton, J. Precategorical acoustic storage (PAS). Perception \& Psychophysics, 1969, 5, 365-373.

Cutting, J. E. Auditory and linguistic processes in speech perception: Inferences from six fusions in dichotic listening. Psychological Review, 1976, 83, 114.140.

DIEHL, R. L. The effects of selective adaptation on the identification of speech sounds. Perception \& Psychophysics, 1975, 17, 48-52.

DiEHL, R. L. Feature analyzers for the phonetic dimension stop vs. continuant. Perception \& Psychophysics, 1976, 19, 267-272.

Diehl, R. L., Elman, J. L., \& McCusker, S. B. Contrast effects in stop consonant identification. Journal of Experimental Psychology: Human Perception and Performance, 1978, 4, 599-609.

Ermas, P. D. The relation between identification and discrimination along speech and non-speech continua. Language and Speech, 1963, 6, 206-217.

Eimas, P. D., CoOper, W. E., \& Corbit, J. D. Some properties of linguistic feature detectors. Perception \& Psychophysics, 1973, 13, 247-252.

Eimas, P. D., \& Corbit, J. D. Selective adaptation of linguistic feature detectors. Cognitive Psychology, 1973, 4, 99-109.

Eimas, P. D., \& MiLlER, J. L. Effects of selective adaptation on the perception of speech and visual patterns: Evidence for feature detectors. In H. L. Pick \& R. D. Walk (Eds.), Perception and experience. New York: Plenum, 1978.

FANT, G. Auditory patterns of speech. In W. Wathen-Dunn (Eds.), Models for the perception of speech and visual form. Cambridge, Mass: MIT Press, 1967.

ForEIT, K. G. Linguistic relativism and selective adaptation for speech: A comparative study of English and Thai. Perception \& Psychophysics, 1977, 21, 347-351.

Fry, D., Abramson, A., Emas, P. D., \& Lugerman, A. M. The identification and discrimination of synthetic vowels. Language and Speech, 1962, 5, 171-189.

Fujisaki, H., \& Kawashima, T. On the modes and mechanisms of speech perception. Annual Report of the Engineering Research Institute, University of Tokyo, 1969, 28, 67-73.

Fujis AKI, H., \& KaWAshima, T. Some experiments on speech perception and a model for the perceptual mechanisms. Annual Report of the Engineering Research Institute, University of Tokyo, 1970, 29, 207-214.

Gerstman, L. J. Classification of self-normalized vowels. IEEE Transactions on Audio and Electroacoustics, 1968, AU-16, 78-80.

Helson, H. Adaptation level theory. New York: Harper \& Row, 1964.

Liberman, A. M., Cooper, F. S., Harrus, K. S., \& MacNeilage, P. F. A motor theory of speech perception. Proceedings of the Speech Communication Seminar, Stockholm, 1962. (Stockholm: Royal Institute of Technology, 1963, D3.)

Liberman, A. M., Cooper, F. S., Harris, K. S., MacNeilage, P. F., \& Studdert-Kennedy, M. Some observations on a model for speech perception. In W. Wathen-Dunn (Ed.), Models for the perception of speech and visual form. Cambridge, Mass: MIT Press, 1967.

Morse, P. A., Kass, J. E., \& Turkienicz, R. Selective adaptation of vowels. Perception \& Psychophysics, 1976, 19, 137-143.

Parducci, A. Range-frequency compromise in judgment. Psychological Monographs, 1963, 77, 2, 1-50.

Parduccr, A. Category judgment: A range-frequency model. Psychological Review, 1965, 72, 407, 418 .

Parducci, A. Contextual effects: A range-frequency analysis. In E. C. Carterette \& M. P. Friedman (Eds.), Handbook of perception (Vol. II). New York: Academic Press, 1975.

Pisoni, D. B. Auditory and phonetic memory codes in the discrimination of consonants and vowels. Perception \& Psychophysics, 1973, 13. 253-260.

PIsonı, D. B. Auditory short-term memory and vowel perception. Memory \& Cognition, 1975, 3, 7-18. (a)

Pisoni, D. B. Dichotic listening and processing phonetic features. In F. Restle, R. M. Shiffrin, N. J. Castellan, H. Lindman, \& D. B. Pisoni (Eds.), Cognitive theory (Vol. I). Hillsdale, N.J: Erlbaum, 1975. (b)

PisonI, D. B., \& SAwusch, J. R. Some stages of processing in speech perception. In A. Cohen \& S. G. Nooteboom (Eds.), Structure and process in speech perception. New York: SpringerVerlag, 1975.

PisonI, D. B., \& TASH, J. B. Auditory property detectors and processing place features in stop consonants. Perception \& Psychophysics, 1975, 18, 401-408.

Restle, F. Assimilation predicted by adaptation-level theory with variable weights. In N. J. Castellan \& F. Restle (Eds.), Cognitive theory (Vol. 3). Hillsdale, N.J: Erlbaum, 1978.

SAWUSCH, J. R. Peripheral and central processes in selective adaptation of place of articulation in stop consonants. Journal of the Acoustical Society of America, 1977, 62, 738-750. (a)

SAwusCh, J. R. Processing of place information in stop consonants. Perception \& Psychophysics, 1977, 22, 417-426. (b)

Sawusch, J. R., \& Pisoni, D. B. Response organization and selective adaptation to speech sounds. Perception \& Psychophysics, $1976,20,413-418$.

Simon, H. J., \& Studdert-Kennedy, M. Selective anchoring and adaptation of phonetic and nonphonetic continua. Journal of the Acoustical Society of A merica, 1978, 64, 1338-1357.

Stevens, K. N., \& Halle, M. Remarks on analysis by synthesis and distinctive features. In W. Wathen-Dunn (Ed.), Models for the perception of speech and visual form. Cambridge, Mass: MIT Press, 1967.

TARTTER, V. C., \& Eimas, P. D. The role of auditory and phonetic feature detectors in the perception of speech. Perception \& Psychophysics, 1975, 18. 293-298.

Thompson, C. L., \& Hollien, H. Some contextual effects on the perception of synthetic vowels. Language \& Speech, 1970, 13, 1-13.

\section{NOTES}

1. All statistical tests, unless otherwise noted, were two-tailed $t$ tests for correlated measures.

2. The significant tests results were: $t(9)=5.00, \mathrm{p}<.001 ; \mathrm{t}(9)$ $=9.29, \mathrm{p}<.001 ; \mathrm{t}(9)=4.44, \mathrm{p}<.002 ;$ and $\mathrm{t}(9)=3.61$, $\mathrm{p}<.01$, for Stimuli 4 through 7 , respectively.

(Received for publication August 31, 1978; revision accepted January $18,1979$. 